\title{
Multitarget Tracking Performance Analysis Using the Non-Credibility Index in the Nonlinear Estimation Framework (NEF) Toolbox
}

\author{
Erik P. Blasch \\ Defence R\&D Canada-Valcartier \\ 2459 Pie-XI Blvd. North \\ Québec City, QC G3J 1X5 \\ erik.blasch@drdc-rddc.gc.ca
}

\author{
Ondřej Straka, Jindřich Duník, Miroslav Šimandl \\ Dept. of Cybernetics, Univ. West Bohemia \\ Univerzini 8, 30614 \\ Pilsen, Czech Republic \\ straka30,dunikj, simandl \{@kky.zcu.cz\}
}

\begin{abstract}
Target tracking, nonlinear control, and fault detection are typically evaluated with only a Root Mean Square (RMS). RMS is an absolute measurement of the system performance and does not provide a statistic as to the tracker, controller, or fault detection algorithmic performance. For this paper, we investigate the noncredibility index (NCI) and average normalized estimation error square (ANEES) for nonlinear estimation for the Kalman Filter $(K F)$, the Central Difference Filter (DD1), the unscented Kalman filter (UKF), and the particle filter $(P F)$. Fault detection and target track performance is dependent on target maneuvers, sensor errors, model parameters, and state estimation which need to be understood relative to the filter performance versus the absolute performance (i.e. root mean square) of the system. Utilizing the developments of the Nonlinear Estimation Framework (NEF) toolbox, we develop methods of nonlinear relative comparison performance between nonlinear filters in a unified scenario.
\end{abstract}

Keywords: Track metrics, RMS, non-credibility index, Relative track metrics, ANEES, PF, UKF, PDAF

\section{Introduction}

In a dynamic targeting scenario, there are host of algorithms that affect performance: sensor registration, measurement-to-track assignment, track-to-track association, sensor management, and ultimately, the user. A key determinant of target-tracking success is being able to choose the right sensor with the correct measurementto-track algorithm. Typically, a track estimation algorithm is evaluated by the absolute position error (i.e. Root Mean Square) [1]. Using the RMS represents the sensoralgorithm performance, as opposed to evaluating the tracker independent of the sensor. In deployable systems, it is important to assess the tracker capability over different operational conditions (target dynamics, sensor quality, and environment obstacles). To evaluate tracker capability independent of operating conditions (OCs), we utilize relative metrics [1].

The goal of target tracking is to associate measurements of moving objects. There are many approaches such as the multiple model estimator (MME)
[2], joint probabilistic data association (JPDAF) [3], jointbelief data association filter (JPBDAF) [4], multiple hypothesis tracker (MHT) [5], interactive multiple model (IMM) [6], variable-structure IMM (VS-IMM) [7], unscented Kalman filter (UKF) [8, 9], particle filter $[10,11,12]$, and the sigma-point KF [13]. Others have used identification information to improve tracker performance $[14,15,16,17,18]$. Other methods explore track information for intent [19], group tracking [20], belief functions[21], and IPDA[22]. Explorations of methods have been applied to simultaneous tracking and identification for radar [23] and electro-optical systems [24]. One difficulty for a user is to determine the best approach for a given scenario such as clutter. To determine the tracker algorithm performance for various OCs, there is a need to study track metrics.

Tracking is important in applications such as robotics, $[25,26]$ and situation awareness [27]. Tracker development includes road constraints [28], nonlinear constraints [29], feature extraction [30], and hyperspectral analysis [31]. Recent advances include awareness for sensor management [32], infrared $\mathrm{L}_{1}$ regularization [33], wide-area sensing [34], and belief-IMM [35] methods.

\begin{tabular}{|l|l|l|l|l|}
\hline Type & Model & Noise & ID Sys. & Ref. \\
\hline IMM & Linear & G & DS & Dezert, 1990 \\
\hline KF & Linear & G & DS/Bayes & Blasch, 1997 \\
\hline MHT & Linear & G & Bayes Net & Fung, 1997 \\
\hline Bootstrap & NL & G & Kin. ID & Salomond, 1997 \\
\hline EKF & NL & G & Mutual Info & Blasch, 1998 \\
\hline UKF & NL & G & None & Julier, 1999 \\
\hline PF & NL & NG & Kinematic & Gordon, 2002 \\
\hline Particles & NL & NG & Kinematic & Angelova, 2004 \\
\hline UPF & NL & NG & PF & Mihaylova, 2005 \\
\hline
\end{tabular}

\subsection{Target Tracking Evaluation}

Drummond investigated various approaches for tracker performance and compiled a list of absolute metrics [36, 37, 38] and Blair for benchmark problems [39]. Evaluation is important for sensor management [40] and sensor sampling [41]. Chong and Mori looked at track association metrics [42]. A third approach is the CramérRao lower bound [43, 44]. Track errors [45], model determination [46], and lack of reference data have been explored [47]. Finally metrics for track and ID were 
developed [48], as well as model-switch for an belief IDaided IMM tracker [49].

One emerging set of metrics from X. Rong Li and coauthors is called relative metrics [1]. Li promotes the usefulness of relative metrics as statistical estimators to isolate tracker performance independent of the data. The relative track metrics include ANEES, Non-Credibility Index, and Credibility Index [1]. The two credibility measures (NCI and ANEES) differ with respect to equitable treatment of optimism versus pessimism. The $\mathrm{NCI}$ is a geometric average, with the mean near the mode. Hence optimism and pessimism are treated equitably. The ANEES is an arithmetic average, and hence severely penalizes optimism.

In data association tracking approaches, typical tracking is provided through position measurements. For linear Gaussian cases, this is acceptable. However, for nonlinear cases the Gaussian assumption is not viable. Of recent interest is the particle filter $(\mathrm{PF})$ method which uses a series of particles to estimate the likelihood of a target location. The sampling approaches approximate the posterior density by a set of samples: the unscented Kalman filter (UKF) uses a small number of deterministically chosen samples, while the particle filter uses a large number of random (Monte Carlo) samples.

We are concerned with assessing track quality and sensor pointing using performance metrics. Many metrics are absolute metrics that are focused on the RMS position error. To better assess how well a nonlinear tracker [24] does based on a given sensor, we focused on the relative track metrics.

Most track methods utilize covariance information to gate measurements and determine tracker quality. Track error might have better representations than just covariance [25]. The goal of this work was to explore the use of the relative metrics to non-linear tracking methods. We chose to explore the use of relative metrics to the linear particle data association filter, suboptimal nonlinear unscented $K F$, and the optimal nonlinear particle filter. Assessing the relative metrics for nonlinear approaches required some modifications to "estimate" the density information. As shown in the paper, useful results were gained in using the relative metrics in assessing tracker quality.

\subsection{Nonlinear Estimation}

Target tracking includes estimation and correlation. To explore non-linear estimation methods, techniques have been developed beyond the extended Kalman Filter (EKF), which linearizes about a point. Šimandl began with advanced point mass functions [50], and Straka et. al., developed PF methods for nonlinear estimation [51, 52,53]. Derivative free methods [54] were developed and applied to fault detection and control [55].

This paper develops the relative metrics for the nonlinear estimation framework (NEF) toolbox. Section 2 describes the NEF toolbox. Section 3 lists estimation metrics. Section 4 and 5 describe linear and nonlinear track estimation. Section 6 and 7 show a performance analysis and draw conclusions.

\section{Nonlinear Estimation Toolbox (NEF)}

The University of West Bohemia, Dept of Cybernetics has developed a nonlinear estimation framework toolbox (NEF) http://nft.kky.zcu.cz/ which was featured in Sysid09 [56]. To support the development of the toolbox for the information fusion community, we are updating the toolbox to include various metrics of analysis. The NEF toolbox (references) features a variety of estimators

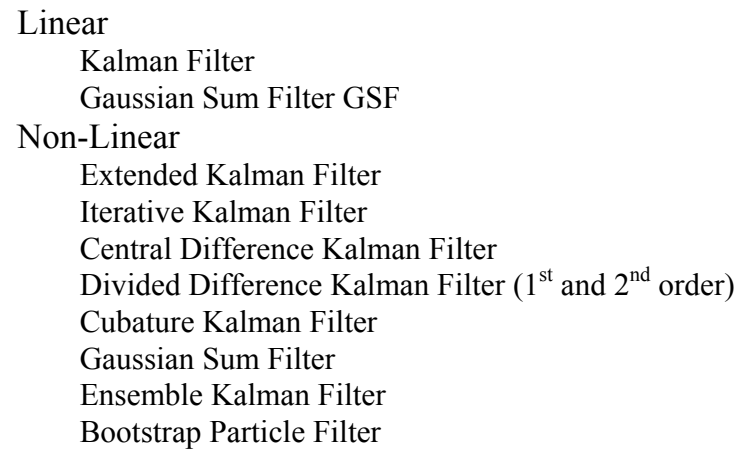

NEF contains a variety of examples including: parameter estimation, track before detect, and fault detection. The examples compare a variety of estimator techniques with traditional RMS and PDF estimation results. Figure 1 overviews the NEF components with the addition of the performance evaluation component described in this paper. Figure 2 shows the model descriptions and function calls.

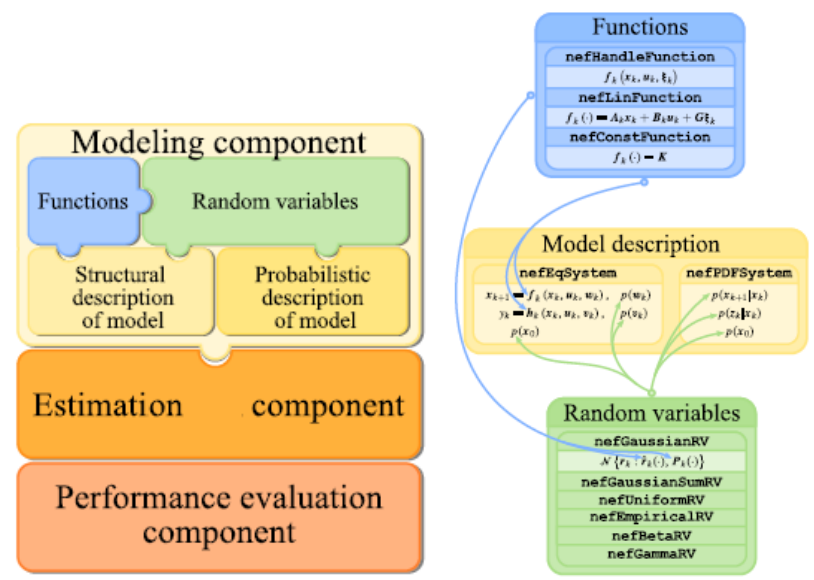

Figure 1. NEF Components. Figure 2. NEF Models.

\section{Tracking and Estimator Metrics}

Tracking methods include many opportunities for analysis. Some metrics are listed below:
Metric
Absolute Track Quality
Description acceleration error
Mean square position, velocity, 
Relative Track Quality

Track Life-Time

Relative Track Life-Time

Track Length

Relative Track Length

Track Purity

Track Coverage

Track Density

Track Continuity
Mean square kinematic error relative to sensor covariance

Total time target is in track

Total time target in track, relative to length of track-lets

Distance over which target is tracked

Distance over which target is tracked relative to maneuverability

Percent of associations of dominant track over lifetime [3]

Area of tracking

Number of targets track per area

Number of individual targets associated with a given track

An objective function for measurement-to-track assignment can be a composite function of the track factors. Blackman [3] has described the use of marginal utility as the assignment objective function. Utility is defined as the ratio of current performance (in the reference application it is tracking performance, measured as estimation error standard deviation) to desired performance. The desired performance is standard criteria established by the tracker. Marginal utility is defined as the difference between current utility and the expected utility that would be achieved if the sensor is allocated to the target. Computation of expected utility requires a model of tracker performance if the sensor is assigned to each candidate target. Thus, we are interested in relative metrics to determine nonlinear estimation of a sensortracker performance model as shown in Figure 3 [57].

\begin{tabular}{|c|c|}
\hline & ABSOLUTE METRICS \\
\hline MSEM & mean squared error matrix \\
\hline RMSE & root mean squared error \\
\hline AEE & average Euclidean error \\
\hline HAE & harmonic average error \\
\hline GAE & geometric average error \\
\hline MEDE & median error \\
\hline \multirow[t]{2}{*}{ MODE } & mode error \\
\hline & RELATIVE METRICS \\
\hline RMSRE & root mean squared relative error \\
\hline ARE & average Euclidean relative error \\
\hline BEEQ & Bayesian estimation error quotient \\
\hline EMER & $\begin{array}{l}\text { estimation error relative to mea- } \\
\text { surement error }\end{array}$ \\
\hline \multicolumn{2}{|r|}{ PERFORMANCE METRICS } \\
\hline NCI & non-credibility index \\
\hline ANEES & $\begin{array}{l}\text { average normalized estimation er- } \\
\text { ror squared }\end{array}$ \\
\hline
\end{tabular}

Figure 3. NEF Metrics.

The Averaged Normalized estimation error squared (ANEES) is defined by [1]:

$$
\text { ANEES }=\frac{1}{N n} \sum_{\mathrm{i}=1}^{\mathrm{N}}\left(\underline{x}_{\mathrm{i}}-\underline{\hat{x}}_{\mathrm{i}}\right)^{\mathrm{T}} \mathbf{P}_{\mathrm{i}}^{-1}\left(\underline{x}_{\mathrm{i}}-\underline{\hat{x}}_{\mathrm{i}}\right)
$$

where $\left(\mathbf{x}_{\mathrm{i}}-\hat{\mathbf{x}}_{\mathrm{i}}\right)$ is the state estimation error, $\mathbf{P}_{\mathrm{i}}$ is the error covariance provided by the estimator in the $\mathrm{i}^{\text {th }}$ run, $n$ is the state dimension, and $N$ is the MC number of runs. If the estimation error and estimated covariance are the same, then the ANEES 1, and the filter is credible [1].
The credibility test is an assessment of the Chi-square capability of the estimator. The determinant is a check for the Non-credibility Index as to whether the sensor system is credible. For an "isotropic" filter, P is proportional to $\Sigma$. In this case, the credibility as defined in Eq. (2) remains to be constant all the time. When the ANEES is outside the $95 \%$ probability interval, the estimator is not credible. For the vector case, the NCI is the sample average $10 \mathrm{Log}$ ${ }_{10}(\rho)$ (analogous to the SNR).

$$
\text { Credibility }=\rho=\frac{\left(\mathbf{x}_{\mathrm{i}}-\hat{\mathbf{x}}_{\mathrm{i}}\right)^{\mathrm{T}} \mathbf{P}_{\mathrm{i}}^{-1}\left(\mathbf{x}_{\mathrm{i}}-\hat{\mathbf{x}}_{\mathrm{i}}\right)}{\left(\mathbf{x}_{\mathrm{i}}-\hat{\mathbf{x}}_{\mathrm{i}}\right)^{\mathrm{T}} \Sigma_{\mathrm{i}}^{-1}\left(\mathbf{x}_{\mathrm{i}}-\hat{\mathbf{x}}_{\mathrm{i}}\right)}
$$

where $\Sigma_{\mathrm{i}}$ is the actual MSE covariance of the sensor.

$$
\begin{aligned}
& \text { Non-Credibility Index }=\frac{10}{\mathrm{~N}} \sum_{\mathrm{i}=1}^{\mathrm{N}} \log 10\left(\rho_{\mathrm{i}}\right) \\
& \text { If } \mathrm{NCI} \gg>0, \mathbf{P}_{\mathrm{i}} \text { is low (optimistic) } \\
& \text { If } \mathrm{NCI}<<0, \mathbf{P}_{\mathrm{i}} \text { is high (pessimistic) }
\end{aligned}
$$

Many estimators / filters provide self assessment of estimation errors - error covariance. However, are the self-assessments (error-covariance) close to the actual MSE, and if so how close? A credibility test determines how much we can trust these self-assessments. An estimator is: Credible if its actual error and selfassessment are statistically equal (e.g. their difference is statistically insignificant). It is Optimistic if its selfassessment is statistically smaller than the actual error and Pessimistic if its self-assessment is statistically larger than the actual error. An estimator is not credible usually because its assumptions / model / approximations are not accurate.

\section{Linear Tracking}

Kalman filters are the baseline for tracking and are optimal if the process and measurement equations are linear and the noise is Gaussian. To enhance the tracker performance, a bank of Kalman filters can be used where different state transition matrices are varied based on the kinematics of the target being tracked. The state and measurement equations for all targets are:

$$
\begin{aligned}
& \underline{\hat{x}}_{\mathrm{t}+1 \mid \mathrm{t}}=F_{\mathrm{t}} \underline{\hat{x}}_{\mathrm{t} \mid \mathrm{t}} \\
& \hat{z}_{\mathrm{t}+1 \mid \mathrm{t}}=H_{\mathrm{t}+1} \underline{\hat{x}}_{\mathrm{t}+1 \mid \mathrm{t}}
\end{aligned}
$$

The covariance of the predicted state is,

$$
P_{\mathrm{t}+1 \mid \mathrm{t}}=F_{\mathrm{t}} P_{\mathrm{t} \mid \mathrm{t}} F_{\mathrm{t}}^{\mathrm{T}}+Q_{\mathrm{t}}
$$

All measurements received from a sensor at time $k$ are then compared to the predicted measurements to ascertain whether or not the measurements fall within the kinematic gate. The validation region is the elliptical region:

$$
V(t, \gamma)=\left\{z:\left[z-\hat{z}_{\mathrm{t}+1 \mid \mathrm{t}}\right]^{\mathrm{T}} S^{-1}{ }_{\mathrm{t}}\left[z-\hat{z}_{\mathrm{t}+1 \mid \mathrm{t}}\right] \leq \gamma\right\}
$$


where $\gamma$ is the gate threshold. Using the gate and the relative metrics we can determine a model mismatch.

\section{Nonlinear Tracking}

\subsection{Suboptimal KF - UKF}

The UKF is a recursive minimum mean square error estimator that often provides an improvement over the extended Kalman filter (EKF) for nonlinear models. The EKF linearizes the nonlinear process and measurement models using the first-order terms of a Taylor series expansion. The UKF, on the other hand, does not approximate the nonlinear process and measurement models. It uses the actual models and instead approximates the distribution of the state variable as a Gaussian. The Gaussian approximation is specified using a minimal set of deterministically chosen samples called sigma points. Each sigma point is independently propagated through the process and measurement models, and the set of propagated sigma points is analyzed to provide a posterior Gaussian approximation.

The nonlinear density and state models are:

$$
\begin{aligned}
& p\left(\underline{x}_{\mathrm{t}} \mid \underline{x}_{\mathrm{t}-1}\right) \\
& \underline{x}_{\mathrm{t}}=f\left(\underline{u}_{\mathrm{t}-1}, \underline{x}_{\mathrm{t}-1}, \underline{v}_{\mathrm{t}-1}\right) \\
& \underline{x}_{\mathrm{t}} \approx f\left(\underline{u}_{\mathrm{t}-1}, \underline{\bar{x}}_{\mathrm{t}-1}, \underline{\bar{v}}\right)+F_{\mathrm{t}}\left(\underline{x}_{\mathrm{t}-1}-\underline{\bar{x}}\right)+G_{\mathrm{t}}\left(\underline{v}_{\mathrm{t}-1}-\underline{\bar{v}}\right)
\end{aligned}
$$

where $\underline{x}_{\mathrm{t}}$ denotes the state of the system at time $t, \underline{u}_{\mathrm{t}} \in \mathfrak{R}^{\mathrm{nu}}$ is some known input, $\underline{v}_{t} \in \Re^{\text {nv }}$ is a noise term with the covariance matrix $\mathbf{Q}, f: \mathfrak{R}^{\mathrm{nx}} \times \mathfrak{R}^{\mathrm{nu}} \times \mathfrak{R}^{\mathrm{nv}} \rightarrow \mathfrak{R}^{\mathrm{nx}}$ is the deterministic nonlinear state transition model and $F_{\mathrm{t}}=$ $\left.\left[\partial f\left(x_{\mathrm{t}}\right) / \partial x_{\mathrm{t}}\right]\right|_{x_{\mathrm{t}}}=\bar{x}_{\mathrm{t}}$ and $G_{\mathrm{t}}=\left[\partial f\left(v_{\mathrm{t}}\right) / \partial v_{\mathrm{t}}\right] \mid v_{\mathrm{t}}=\bar{v}_{\mathrm{t}}$ are the Jacobians of the state model linearized around the reference value $\underline{\bar{x}}_{\mathrm{t}}$ and $\underline{\bar{v}}_{\mathrm{t}}$, respectively.

The nonlinear measurement model is

$$
\begin{aligned}
& p\left(\underline{y}_{\mathrm{t}} \mid \underline{x}_{\mathrm{t}-1}\right) \\
& \underline{y}_{\mathrm{t}}=h\left(\underline{u}_{\mathrm{t}}, \underline{x}_{\mathrm{t}}, \underline{n}_{\mathrm{t}}\right) \\
& \underline{y}_{\mathrm{t}} \approx f\left(\underline{u}_{\mathrm{t}}, \underline{\bar{x}}_{\mathrm{t}}, \underline{\bar{n}}\right)+H_{\mathrm{t}}\left(\underline{x}_{\mathrm{t}}-\underline{\bar{x}}\right)+L_{\mathrm{t}}\left(\underline{n}_{\mathrm{t}}-\underline{\bar{n}}\right)
\end{aligned}
$$

where $\underline{y}_{\mathrm{t}} \in \mathfrak{R}^{\mathrm{ny}}$ is the measurement vector, $\underline{n}_{\mathrm{t}} \in \mathfrak{R}^{\mathrm{nn}}$ the measurement noise with covariance matrix $\mathbf{R}$, which is assumed to be independent of $\underline{v}_{\mathrm{t}}, h: \mathfrak{R}^{\mathrm{nx}} \times \mathfrak{R}^{\mathrm{nu}} \times \mathfrak{R}^{\mathrm{nn}} \rightarrow$ $\mathfrak{R}^{\mathrm{nu}}$ is the deterministic nonlinear measurement, $H_{\mathrm{t}}=$ $\left.\left[\partial h\left(x_{\mathrm{t}}\right) / \partial x_{\mathrm{t}}\right]\right|_{x_{\mathrm{t}}}=\bar{x}_{\mathrm{t}}$ and $L_{\mathrm{t}}=\left[\partial h\left(n_{\mathrm{t}}\right) / \partial n_{\mathrm{t}}\right] n_{n_{\mathrm{t}}}=\bar{n}_{\mathrm{t}}$ are the Jacobians of the measurement model linearized around the reference values of $\underline{\underline{x}}_{\mathrm{t}}$ and $\underline{\bar{n}}$, respectively.

For the UKF, consider a random variable $\underline{x} \in \mathfrak{R}^{\mathrm{n}} \sim$ $\mathcal{N}\left(\underline{m}_{\mathrm{x}}, \mathbf{P}_{\mathrm{x}}\right)$. The covariance matrix is scaled up by a factor $(\mathrm{n}+\kappa)$, thus spreading the standard deviation by a factor of $\gamma=\sqrt{\mathrm{n}+\kappa}$. Now factorize the covariance matrix by the
Cholesky decomposition $\sqrt{\mathbf{P}_{\mathrm{x}}}$. Construct $2 n+1$ sigma points around the mean vector $\mathrm{m}$ with the scaled spread square $\operatorname{root} \gamma \sqrt{\mathbf{P}_{\mathrm{x}}}$ as :

$$
\mathcal{X}=\left[\underline{m}_{\mathrm{x}}, \underline{m}_{\mathrm{x}}+\gamma \sqrt{\mathbf{P}_{\mathrm{x}}}, \underline{m}_{\mathrm{x}}-\gamma \sqrt{\mathbf{P}_{\mathrm{x}}}\right]
$$

Let $[X]_{\mathrm{i}}$ be the $\mathrm{i}^{\text {th }}$ column of $\mathcal{X}$ (i.e. the $\mathrm{i}^{\text {th }}$ sigma point vector with the count starting from 0 ). Propagating it through the nonlinear function $\underline{y}=f(\underline{x})$ produces the posterior sigma point vectors as $[\mathcal{Y}]_{\mathrm{i}}$ for $i=0, \ldots, 2 n$. The mean and covariance are calculated from the weighted mean and covariance as

$$
\begin{aligned}
& \underline{m}_{\mathrm{y}}=\sum_{\mathrm{i}=0}^{2 \mathrm{n}} \mathrm{W}_{\mathrm{i}}{ }^{\mathrm{m}}[\mathcal{Y}]_{\mathrm{i}} \\
& \left.\left.\mathrm{P}_{\mathrm{yy}}=\sum_{\mathrm{i}=0}^{2 \mathrm{n}} \mathrm{W}_{\mathrm{i}}{ }^{\mathrm{c}}\left([\mathcal{Y}]_{\mathrm{i}}-\underline{m}_{\mathrm{y}}\right)[\mathcal{Y}]_{\mathrm{i}}-\underline{m}_{\mathrm{y}}\right)\right)^{\mathrm{T}} \\
& \left.\left.\mathrm{P}_{\mathrm{xy}}=\sum_{\mathrm{i}=0}^{2 \mathrm{n}} \mathrm{W}_{\mathrm{i}}{ }^{\mathrm{c}}\left([\mathcal{X}]_{\mathrm{i}}-\underline{m}_{\mathrm{x}}\right)[\mathcal{Y}]_{\mathrm{i}}-\underline{m}_{\mathrm{y}}\right)\right)^{\mathrm{T}}
\end{aligned}
$$

where $\mathrm{W}_{\mathrm{i}}^{\mathrm{m}}$ and $\mathrm{W}_{\mathrm{i}}^{\mathrm{c}}$ are the constant weights for the mean and covariance, given by

$$
\begin{aligned}
& \mathrm{W}_{0}{ }^{\mathrm{m}}=\frac{\lambda}{\lambda+n} ; \quad \mathrm{W}_{\mathrm{i}}^{\mathrm{m}}=\frac{1}{2(\lambda+n)} ; \mathrm{W}_{\mathrm{i}}{ }^{\mathrm{c}}=\mathrm{W}_{\mathrm{i}}^{\mathrm{m}} \\
& \mathrm{W}_{0}{ }^{\mathrm{c}}=\frac{\lambda}{\lambda+n}+\left(1-\alpha^{2}+\beta\right) ; i=1, . ., 2 n .
\end{aligned}
$$

where $\lambda=\alpha^{2}(\mathrm{n}+\kappa)$. The parameter $\kappa \geq 0$ is chosen to ensure the positive-definiteness of the covariance matrix, which defaults to zero. The parameter $0 \leq \alpha \leq<1$ controls the size of the sigma point distribution (probabilistic spread in terms of covariance) and takes a small number to avoid sampling non-local effects when non-linearities are strong. $\beta$ is a weighting term used to incorporate any knowledge of higher order moments of the distribution. For a Gaussian, the optimal choice is $\beta=$ 0 [13].

The UKF is a scaled unscented transformation (SUT) [9] to recursive minimum mean-square estimator (MMSE) estimation. The propagation of a random variable through a nonlinear function with the SUT (as in the UKF) leads to a recursive estimation steps for the UKF.

Step 1 - Construct an augmented state by concatenating the original state with the process noise and measurement noise.

Step 2 - Calculate the covariance matrix for the augmented state and select the sigma points for the augmented state.

Step 3 - Conduct time-update by propagating sigma points through exact nonlinear process and measurement equations.

Step 4 - Calculate covariance matrix of predicted measurements per sigma points $\left(\mathbf{P}_{\mathrm{YY}}\right)$ and cross-covariance matrix between predicted measurements and augmented state $\left(\mathbf{P}_{\mathrm{XY}}\right)$.

Step 5 - Conduct the measurement update using the Kalman filter gain as $\mathcal{K}_{\mathrm{k}}=\mathbf{P}_{\mathrm{XY}}\left(\mathbf{P}_{\mathrm{YY}}\right)^{-1}$. 
Table 1 lists the algorithm that updates the mean and covariance of the Gaussian approximation to the posterior distribution of the state. As shown, there is no explicit calculation of Jacobians or Hessians. Since the covariance matrix can be expressed recursively in the square-root form, not only does the UKF outperform the EKF in accuracy and robustness, it does so at no extra computational cost. When the process and measurement noise terms are purely additive, there is no need to augment the system state with noise, further reducing the computational complexity. Both the EKF and UKF reply on the Gaussian assumption and its approximation. The particle filtering method described next; however, does not require this assumption. But it has problems of its own.

Table 1. The Unscented Kalman Filter [58]

\begin{tabular}{|c|c|}
\hline Concatenation: & $\begin{array}{l}\text { Augment the state vector to include noise terms: } \\
\underline{x}_{t}^{a}=\left[\underline{x}_{t}^{T} \underline{v}_{t}^{T} \underline{n}_{t}^{T}\right]^{T}, n_{a}=n_{x}+n_{\mathrm{v}}+n_{n}\end{array}$ \\
\hline Initialization: & $\begin{array}{l}\underline{\bar{x}}_{0}=E\left\{\underline{x}_{0}\right\}, P_{0}=E\left\{\left(\underline{x}_{0}-\bar{x}_{0}\right)\left(\underline{x}_{0}-\underline{x}_{0}\right)^{T}\right\} \\
\underline{\bar{x}}_{0}^{a}=E\left\{\underline{x}_{0}^{a}\right\}=\left[\underline{x}_{0}^{a}, 0,0\right], P_{0}^{a}=E\left\{\left(\underline{x}_{0}^{a}-\underline{x}_{0}^{a}\right)\left(\underline{x}_{0}^{a}-\underline{x}_{0}^{a}\right)^{T}\right\}=\operatorname{diag}\left\{P_{0}, Q, R\right\}\end{array}$ \\
\hline \multicolumn{2}{|l|}{ Recursive over $\mathrm{t}$ : } \\
\hline Calculate Sigma Points & $\begin{array}{l}\mathrm{X}_{t-1}^{a}=\left[\mathrm{X}_{0, t-1}^{a}, \mathrm{X}_{1, t-1}^{a}, \ldots, \mathrm{X}_{2 n a, t-1}^{a}\right] \text { where } \\
\mathrm{X}_{0, t-1}^{a}=\bar{x}_{t-1}^{a}, W_{0}^{(m)}=\lambda\left(n_{x}+\lambda\right), W_{0}^{(c)}=\lambda\left(n_{\mathrm{x}}+\lambda\right)+\left(1-\alpha^{2}+\beta\right) \\
\mathrm{X}_{i, t-1}^{a}=\bar{x}_{t-1}^{a}+\left[\sqrt{\left(n_{a}+\kappa\right) P_{t-1}^{a}}\right]_{b} i=1, \ldots, n_{a} \\
\mathrm{X}_{i, t-1}^{a}=\bar{x}_{t-1}^{a}-\left[\sqrt{\left(n_{a}+\kappa\right) P_{t-1}^{a}}\right]_{b} i=n_{a}+1, \ldots, 2 n_{a} \\
W_{i}^{(m)}=W_{i}^{(c)}=1 /\left(2\left(n_{x}+\lambda\right)\right), i=1, \ldots, 2 n_{a}\end{array}$ \\
\hline Time Update & 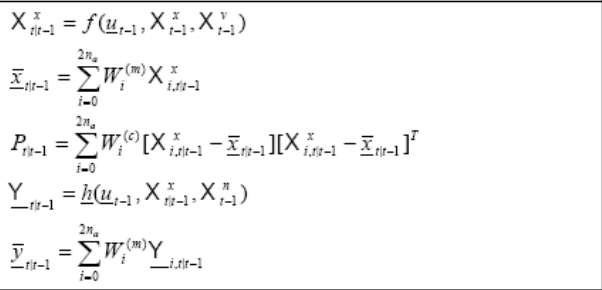 \\
\hline Measurement Update & 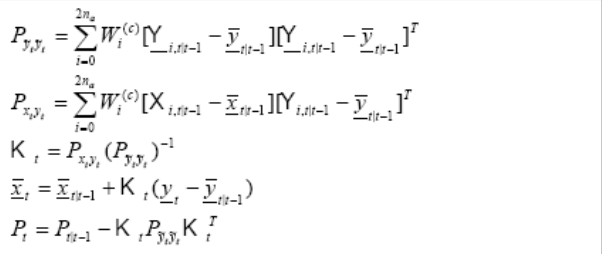 \\
\hline
\end{tabular}

\subsection{Optimal Nonlinear Filter - PF}

Classical Monte Carlo methods for dynamic systems, such as particle filters, are capable of tracking complex nonlinear systems with noisy measurements. The problem is that estimates from a particle filter tend to have a high variance for small sample sets. Using large sample sets is computationally expensive and defeats the purpose.

Particle filters have been extensively used for Bayesian state estimation in nonlinear systems with noisy measurements. They approximate the probability distribution with a set of samples or particles. Particle filters have a number of characteristics that make them attractive: they are nonparametric (can represent arbitrary distributions), can handle hybrid state spaces, can handle noisy sensing and motion, and can easily be balanced to meet performance objectives where the number of particles (hence, estimation accuracy) can be adjusted to match available computation. The design of a particle filter is based on four basic ideas, which are discussed below.

\subsection{Discrete Approximation of PDF}

The first idea is the approximation of a continuous support distribution $p\left(x_{0: \mathrm{t}} \mid y_{1: \mathrm{t}}\right)$ by $N$ discrete samples $\mathrm{x}_{0: \mathrm{t}}$, "randomly" drawn from the distribution $p\left(\mathrm{x}_{0: \mathrm{t}} \mid \mathrm{y}_{1: \mathrm{t}}\right)$, for $\mathrm{i}=1, \ldots, N$ :

$$
p\left(x_{0: \mathrm{t}} \mid y_{1: \mathrm{t}}\right) \cong \frac{1}{N} \sum_{\mathrm{i}=1}^{\mathrm{N}} \delta\left(\mathrm{x}_{0: \mathrm{t}}-\mathrm{x}_{0: \mathrm{t}}^{(\mathrm{i})}\right)
$$

where the subscript " $0: t$ " or " $1: t$ " indicates the observation interval from 0 or 1 to $\mathrm{t}$ and $\delta(\bullet)$ is the Dirac delta function. This is the so-called Monte Carlo method. With this approximation, the computation of expectation of any function of $\mathrm{x}_{0: \mathrm{t}}, \mathrm{g}(\bullet)$, is reduced from a complicated integration to a simple summation as:

$$
\begin{gathered}
E\left\{g_{\mathrm{t}}\left(x_{0: \mathrm{t}}\right)\right\}=\int g_{\mathrm{t}}\left(x_{0: \mathrm{t}}\right) p\left(x_{0: \mathrm{t}} \mid y_{1: \mathrm{t}}\right) \mathrm{d} x_{0: \mathrm{t}} \\
\cong \frac{1}{N} \sum_{\mathrm{i}=1}^{\mathrm{N}} \mathrm{g}_{\mathrm{t}}\left(\mathrm{x}_{0: \mathrm{t}}{ }^{(\mathrm{i})}\right)
\end{gathered}
$$

\subsection{Importance Sampling}

The second idea is the importance sampling. In the estimation problem, the posterior distribution $p\left(x_{0: \mathrm{t}} \mid y_{1: \mathrm{t}}\right)$ is in fact what we want to estimation from data, thus not available for sampling directly. One way to get around this is to approximate the expectation over the unknown distribution $p\left(x_{0: \mathrm{t}} \mid y_{1: \mathrm{t}}\right)$ by another expectation taken over a known easy-to-sample distribution $q\left(x_{0: \mathrm{t}} \mid y_{1: \mathrm{t}}\right)$, called importance function, also known as proposal distribution (which we will use interchangeably):

$$
\begin{aligned}
& E\left\{g_{\mathrm{t}}\left(x_{0: \mathrm{t}}\right)\right\}=\int g_{\mathrm{t}}\left(x_{0: \mathrm{t}}\right) \frac{p\left(x_{0: \mathrm{t}} \mid y_{1: \mathrm{t}}\right)}{q\left(x_{0: \mathrm{t}} \mid y_{1: \mathrm{t}}\right)} q\left(x_{0: \mathrm{t}} \mid y_{1: \mathrm{t}}\right) \mathrm{d} x_{0: \mathrm{t}} \\
& =\int g_{\mathrm{t}}\left(x_{0: \mathrm{t}}\right) \frac{p\left(y_{1: \mathrm{t}} \mid x_{0: \mathrm{t}}\right) p\left(x_{0: \mathrm{t}}\right) / p\left(y_{1: \mathrm{t}}\right)}{q\left(x_{0: \mathrm{t}} \mid y_{1: \mathrm{t}}\right)} q\left(x_{0: \mathrm{t}} \mid y_{1: \mathrm{t}}\right) \mathrm{d} x_{0: \mathrm{t}} \\
& =\int g_{\mathrm{t}}\left(x_{0: \mathrm{t}}\right) \frac{w_{\mathrm{t}}\left(x_{0: \mathrm{t}}\right)}{p\left(y_{1: \mathrm{t}}\right)} q\left(x_{0: \mathrm{t}} \mid y_{1: \mathrm{t}}\right) \mathrm{d} x_{0: \mathrm{t}}
\end{aligned}
$$

where the variable $w_{\mathrm{t}}\left(x_{0: \mathrm{t}}\right)$ is the un-normalized importance weight defined by:

$$
w_{\mathrm{t}}\left(x_{0: \mathrm{t}}\right)=\frac{w_{\mathrm{t}}\left(x_{0: \mathrm{t}}\right)}{p\left(y_{1: \mathrm{t}}\right)}
$$

The probability density function $p\left(\begin{array}{lll}y & 1: & \mathrm{t}\end{array}\right)$ in the denominator can be evaluated as:

$p\left(y_{1: \mathrm{t}}\right)=\int p\left(y_{1: \mathrm{t}} \mid x_{0: \mathrm{t}}\right) p\left(x_{0: \mathrm{t}}\right) \frac{q\left(x_{0: \mathrm{t}} \mid y_{1: \mathrm{t}}\right)}{q\left(x_{0: \mathrm{t}} \mid y_{1: \mathrm{t}}\right)} \mathrm{d} x_{0: \mathrm{t}}$ 


$$
\begin{aligned}
& =\int w_{\mathrm{t}}\left(x_{0: \mathrm{t}}\right) q\left(x_{0: \mathrm{t}} \mid y_{1: \mathrm{t}}\right) \mathrm{d} x_{0: \mathrm{t}} \\
& =E_{\mathrm{q}}\left\{w_{\mathrm{t}}\left(x_{0: \mathrm{t}}\right)\right\}
\end{aligned}
$$

where $E_{\mathrm{q}}\{\bullet\}$ is the expectation taken over the importance function or proposal distribution $q\left(x_{0: \mathrm{t}} \mid y_{1: \mathrm{t}}\right)$.

Following Eq. (20), $p\left(y_{1: \mathrm{t}}\right)$ can be taken out of the integral of Eq. (18), still in the denominator, though. By consequence, the numerator of Eq. (18) can be evaluated over $q\left(x_{0: \mathrm{t}} \mid y_{1: \mathrm{t}}\right)$ in the same manner, leading to $E_{\mathrm{q}}\left\{g_{\mathrm{t}}(x\right.$ $\left.0: t) w_{\mathrm{t}}\left(x_{0: \mathrm{t}}\right)\right\}$.

When these expectations are approximated as in Eq. (16) by drawing $\mathrm{N}$ samples from the importance function (proposal distribution) $q\left(x_{0: \mathrm{t}} \mid y_{1: \mathrm{t}}\right)$, we have:

$$
\begin{aligned}
& E\left\{g_{\mathrm{t}}\left(x_{0: \mathrm{t}}\right)\right\}=\frac{E_{\mathrm{q}}\left\{g_{\mathrm{t}}\left(x_{0: \mathrm{t}}\right) w_{\mathrm{t}}\left(x_{0: \mathrm{t}}\right)\right\}}{E_{\mathrm{q}}\left\{w_{\mathrm{t}}\left(x_{0: \mathrm{t}}\right)\right\}}
\end{aligned}
$$

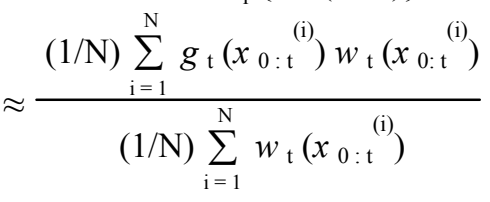

$$
\begin{aligned}
& =\sum_{\mathrm{i}=1}^{\mathrm{N}} g_{\mathrm{t}}\left(x_{0: \mathrm{t}^{(\mathrm{i})}}\right) \frac{(1 / \mathrm{N}) w_{\mathrm{t}}\left(x_{\left.0: \mathrm{t}^{(\mathrm{i})}\right)}\right.}{(1 / \mathrm{N}) \sum_{\mathrm{j}=1}^{\mathrm{N}} w_{\mathrm{t}}\left(x_{\left.0: \mathrm{t}^{(\mathrm{i})}\right)}\right.} \\
& =\sum_{\mathrm{i}=1}^{\mathrm{N}} g_{\mathrm{t}}\left(x_{0: \mathrm{t}^{(\mathrm{i})}}\right) \widetilde{w}_{\mathrm{t}}\left(x_{\mathrm{t}}^{(\mathrm{i})}\right) \\
& \widetilde{w}_{\mathrm{t}}{ }^{(\mathrm{i})}=\frac{w_{\mathrm{t}}{ }^{(\mathrm{i})}}{\sum_{\mathrm{j}=1}^{\mathrm{N}} w_{\mathrm{t}}{ }^{(\mathrm{j})}}
\end{aligned}
$$

The normalized importance weight $\widetilde{w}_{\mathrm{t}}{ }^{(\mathrm{i})}$ is used in the last equality of Eq. (21) with $\sum_{\mathrm{i}=1}^{\mathrm{N}} \widetilde{w}_{\mathrm{t}}{ }^{(\mathrm{i})}=1$.

\subsection{Sequential Importance Sampling (SIS)}

The third idea is sequential importance sampling (SIS). Under the assumptions that the underlying state corresponds to a Markov process [i.e., $p\left(x_{0: \mathrm{t}}\right)=\Pi^{\mathrm{t}}{ }_{\mathrm{j}=1}$ $\left.p\left(x_{\mathrm{j}} \mid x_{\mathrm{j}}-1\right)\right]$, the observations are conditionally independent given the state [i.e., $p\left(y_{1: \mathrm{t}} \mid x_{0: \mathrm{t}}\right)=\Pi^{\mathrm{t}} \mathrm{j=1}$ $\left.p\left(x_{\mathrm{j}} \mid x_{\mathrm{j}}\right)\right]$, and the importance function or proposal distribution is factorable [i.e., $q\left(x_{0: \mathrm{t}} \mid y_{1: \mathrm{t}}\right)=q\left(x_{0: \mathrm{t}-1} \mid y\right.$ $1: \mathrm{t}-1) q\left(x_{\mathrm{t}}\left|x_{0: \mathrm{t}-1}\right| y_{1: \mathrm{t}-1}\right)$ according to the Bayes' rule), then the un-normalized importance weight can be estimated recursively as:

$$
\begin{aligned}
w_{\mathrm{t}}\left(x_{0: \mathrm{t}}\right) & =\frac{\left.p\left(x_{1: \mathrm{t}-1} \mid x_{0: \mathrm{t}-1}\right) p\left(y_{\mathrm{t}} \mid x_{\mathrm{t}}\right)\right] p\left(x_{0: \mathrm{t}-1}\right) p\left(x_{\mathrm{t}} \mid x_{\mathrm{t}-1}\right)}{q\left(x_{0: \mathrm{t}-1} \mid y_{1: \mathrm{t}-1}\right) q\left(x_{\mathrm{t}}\left|x_{0: \mathrm{t}-1}\right| y_{1: \mathrm{t}-1}\right)} \\
& =w_{\mathrm{t}-1}\left(x_{0: \mathrm{t}-1}\right) \frac{p\left(y_{\mathrm{t}} \mid x_{\mathrm{t}}\right) p\left(x_{\mathrm{t}} \mid x_{\mathrm{t}-1}\right)}{q\left(x_{\mathrm{t}}\left|x_{0: \mathrm{t}-1}\right| y_{1: \mathrm{t}-1}\right)}
\end{aligned}
$$

Eq. (23) provides a mechanism to sequentially update the importance weight given the conditional proposal distribution $q\left(x_{\mathrm{t}}\left|x_{0: \mathrm{t}-1}\right| y_{1: \mathrm{t}-1}\right)$. Indeed, we can sample from this proposal distribution (i.e., generate $\mathrm{N}$ discrete samples $x_{\mathrm{t}}{ }^{(\mathrm{i})}$ according to $q\left(x_{\mathrm{t}}\left|x_{0: \mathrm{t}-1}\right| y_{1: \mathrm{t}-1)}\right)$ and evaluate the likelihood and transition probabilities [ i.e., $p\left(y_{\mathrm{t}} \mid x_{\mathrm{t}}\right)$ and $p\left(x_{\mathrm{t}} \mid x_{\mathrm{t}-1}\right)$ given by the process and measurement models of Eqs. (1a) and (2a), respectively) for these samples. Doucet et al. [59] show that the proposal distribution is the "optimal" proposal distribution, that is, the distribution that minimizes the variance of the importance weights conditioned on $x t_{t}-1$ and $y_{\mathrm{t}}$. Table 2 lists the algorithm for a generic particle filter with additional steps discussed below.

\subsection{Resampling and Diversification}

For any practical implementation, the number of samples that can be drawn from a distribution is limited. As such, the choice of importance function or proposal distribution becomes critical, creating other issues impeding the success of a particle filter.

An easy choice of importance function for a process model with additive Gaussian noise is the transition prior:

$$
\begin{aligned}
& q\left(x_{\mathrm{t}}\left|x_{0: \mathrm{t}-1}\right| y_{1: \mathrm{t}-1}\right) \approx p\left(x_{\mathrm{t}} \mid x_{\mathrm{t}-1}\right) \\
& \sim \mathcal{N}\left\{f\left(u_{\mathrm{t}-1}, x_{\mathrm{t}-1}, 0\right), Q_{\mathrm{t}-1}\right\}
\end{aligned}
$$

\begin{tabular}{|c|c|}
\hline Initialization: $\mathrm{t}=0$ & Draw the initial state $x_{0}^{(i)}$ from the prior $p\left(x_{0}\right), i=1, \ldots, N$ \\
\hline \multicolumn{2}{|l|}{ For $\mathrm{t}=1,2$, } \\
\hline \multirow[t]{3}{*}{ Importance Sampling } & Sample $\hat{x}_{t}^{(i)} \sim q\left(x_{t} \mid x_{0: t-1}^{(i)}, y_{1: t}\right)$ and set $\hat{x}_{0 t}^{(i)}=\left[\hat{x}_{0: t-1}^{(0)}, \hat{x}_{t}^{(i)}\right]$ for $i=1, \ldots, N$ \\
\hline & $\begin{array}{l}\text { Evaluate the unnormalized importance weight: } \\
w_{t}^{(i)}=w_{t-1}^{(i)} \frac{p\left(y_{t} \mid \hat{x}_{t}\right) p\left(\hat{x}_{t} \mid \hat{x}_{t-1}\right)}{q\left(\hat{x}_{t}^{(i)} \mid \hat{x}_{t-1}^{(n}, y_{1 z}\right)} \text { for } i=1, \ldots, N\end{array}$ \\
\hline & $\begin{array}{l}\text { Normalize the importance weights: } \\
\widetilde{w}_{t}^{(i)}=w_{t}^{(i)}\left[\sum_{i=1}^{N} w_{t}^{(i)}\right]^{-1} \text { for } i=1, \ldots, N\end{array}$ \\
\hline \multirow[t]{2}{*}{ Selection (Resampling): } & $\begin{array}{l}\text { Multiply (or suppress) samples } \hat{x}_{t}^{(i)} \text { with high (or low) importance weights } \widetilde{w}_{t}^{(i)} \text { to obtain } \\
\text { N random samples } x_{0 t}^{(t)} \text { approximately distributed according to } p\left(x_{0 t}^{(i)} \mid y_{i t}\right)\end{array}$ \\
\hline & Set $w_{t}^{(l)}=\widetilde{w}_{t}^{(t)}=1 / \mathrm{N}$ for $\mathrm{i}=1, \ldots, \mathrm{N}$ \\
\hline \multirow[t]{3}{*}{ Output: } & The output of the algorithm is a set of sampies $\hat{x}_{0 i}^{(i)}$ with weights $\widetilde{w}_{t}^{(i)}=1 / \mathrm{N}$ for $i=1, \ldots, N$ \\
\hline & $\begin{array}{l}\text { The samples can be used to approximate the posterior distribution as } \\
p\left(x_{0 t} \mid y_{1 t}\right) \cong \hat{p}\left(x_{0 t} \mid y_{1 t}\right)=\frac{1}{N} \sum_{i=1}^{N} \delta\left(x_{0 t}-\hat{x}_{0: t}^{(i)}\right)\end{array}$ \\
\hline & $\begin{array}{l}\text { The samples can also be used to calculate expectation for appropriate functions as: } \\
E\left\{g_{t}\left(x_{0 t}\right)\right\}=\int g_{t}\left(x_{0 t}\right) p\left(x_{0 t} \mid y_{1 t}\right) d x_{0 t} \cong \frac{1}{N} \sum_{i=1}^{N} g_{t}\left(\hat{x}_{0 t}^{(i)}\right) \\
\text { which is the marginal conditional mean of } x_{0 t} \text { when } g_{t}\left(x_{0 t}\right)=x_{0 t} \text { and the marginal } \\
\text { conditional covariance of } x_{0 t} \text { when } g_{t}\left(x_{0 t}\right)=x_{0 t} x_{0: t}^{T}-E_{p\left(x_{t} \mid y_{t}\right)}\left\{x_{0 t}\right\} E_{p\left(x_{t} \mid y_{t}\right)}^{T}\left\{x_{0 t}\right\} .\end{array}$ \\
\hline
\end{tabular}

However, this proposal distribution does not incorporate the latest data available and it runs the risk to deplete the samples in the sense that after a few iterations, one of the normalized importance weights tends to 1 while the remaining weights tend to 0 . This effectively removes a large number of samples from the sample set because their importance weights are numerically insignificant.

Table 2. Generic Particle Filter [58]

To avoid degeneracy, the fourth idea is importance resampling, also called selection, in which samples with low importance weights are eliminated while samples with high importance are multiplied keeping the total population of samples at the same level. Techniques for resampling include sampling importance resampling 
(SIR), residual resampling, and minimum variance sampling.

Since the selection step favors the creation of multiple copies of the "fittest" particles (thus allows us to track the updated distributions), many "unfit" particles may end up with few or none copies, leading to sample impoverishment. To solve this problem, an additional step is therefore needed to introduce the sample diversification after the selection step without affecting the validity of the approximation. A brute force approach would increase the number of samples. But a refined technique is to implement a Markov chain Monte Carlo (MCMC) step, which moves new particles to areas of more interest in the state space by applying a Markov chain transition kernel. Figure 3 illustrates this process.

\subsection{Formulation for Classical Particle Filter (CPF)}

1) Draw $\mathrm{N}$ particles $x_{\mathrm{t}}{ }^{(\mathrm{i})}=1, . ., \mathrm{n}$ based on the previous posterior, as follows:

$$
\hat{x}_{1: \mathrm{t}}{ }^{(\mathrm{i})} \sim p\left(\hat{x}_{\mathrm{t}}{ }^{(\mathrm{i})}, x_{1: \mathrm{t}-1}{ }^{(\mathrm{i})}\right)
$$

2) Assign/update importance weight, $w_{\mathrm{t}}$, to each particle:

$$
w_{\mathrm{t}}^{(\mathrm{i})} \sim p\left(y_{\mathrm{t}} \mid x_{\mathrm{t}}{ }^{(\mathrm{i})}\right)
$$

3) Resample, i.e., draw $\mathrm{N}$ particles from the weighted particle set with a probability proportional to the weights (26). Assign the particle set to $x_{1: \mathrm{t}}{ }^{\text {(i) }}$ with $w_{\mathrm{t}}{ }^{\text {(i) }}=1$.

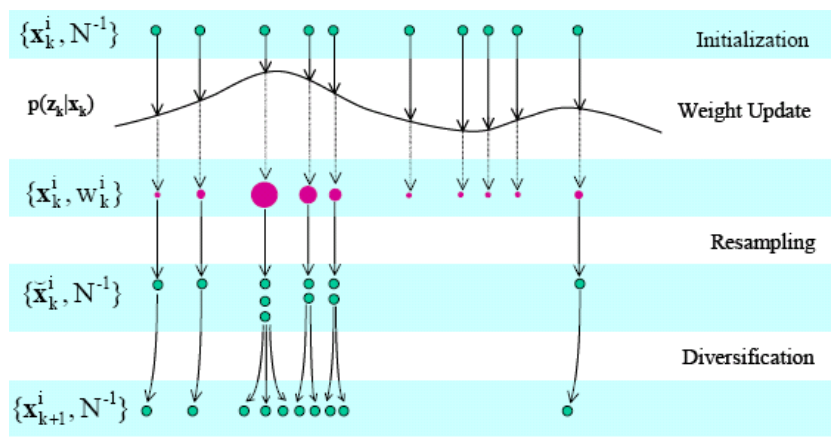

Figure 4. Re-sampling/selection and diversification

Particle filters have computational and representational advantages over other Bayesian techniques. The main problem is that a large number of particles are often needed to maintain a reasonable approximation of the state probability distribution to detect rapid maneuvers which requires maintaining and updating large numbers of particles. This is typically not practical due to limited computation. However, a small particle sets do not provide reasonable approximations because they are unlikely to represent quick maneuver changes and their estimates are likely to have a high variance. The balance between the number of particles and tracker performance is assessed using the NCI estimator.
To overcome such problems, the particle filter may be combined with an EKF or UKF in a hybrid manner [58]. To address the sample size and tracker performance, we use the relative metrics by approximating $\mathbf{P}$ in Eq. (1-3) as a function of the particles.

\section{Relative Performance Analysis}

For this analysis, we first developed a toolbox of performance evaluation methods [57]. Here, we compared the KF, DD1, UKF, and PF using the relative metrics ANESS and NCI versus RMS. The scenario is presented in Figure 4 which includes long-run target maneuvering scenario to assess the difficulty of a tracker losing track. In the investigation the number of particles was varied between 200 and 5000 .

Figure 5 presents the absolute metrics of the trackers. These absolute metrics mask the performance of the tracker relative to the data quality. Thus, we explored methods that look at the estimation quality of the tracker robustness.
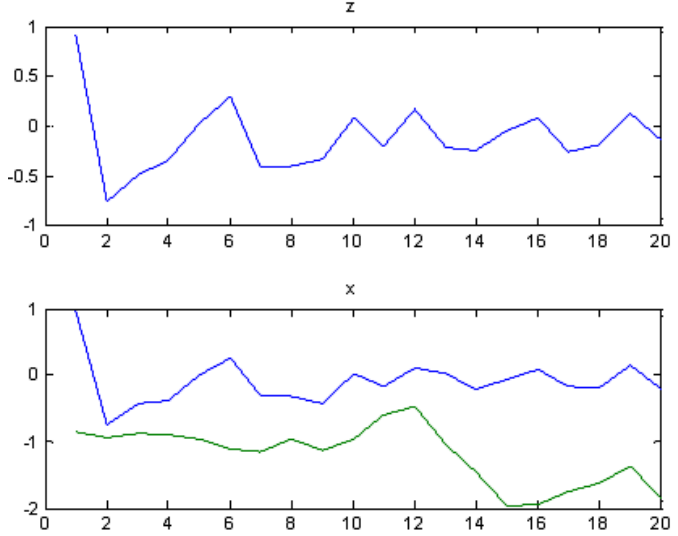

Figure 5. Scenario
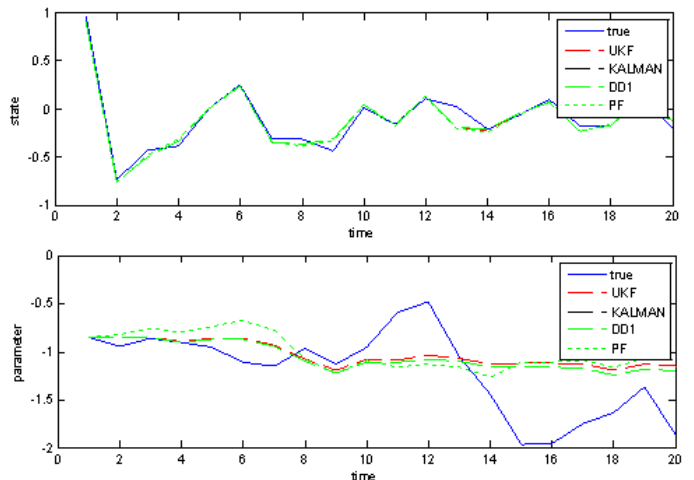

Figure 6. Filter Comparisons

\begin{tabular}{|c|c|c|}
\hline Stats : & MSEM & Time \\
\hline UKF & 0.086898 & 5.257234 \\
\hline DD1 & 0.080364 & 4.820431 \\
\hline KF: & 0.080351 & 4.617630 \\
\hline PF : & 0.104602 & 20.077329 \\
\hline
\end{tabular}

Table 1: Computing statistics of the obtained results 
The Non-Credibility Index using a Monte Carlo Analysis of 1.00 Runs Using the DD1 Process Noise Variance $(Q)=0$ Measurement Noise Variance $(R)=0.01$

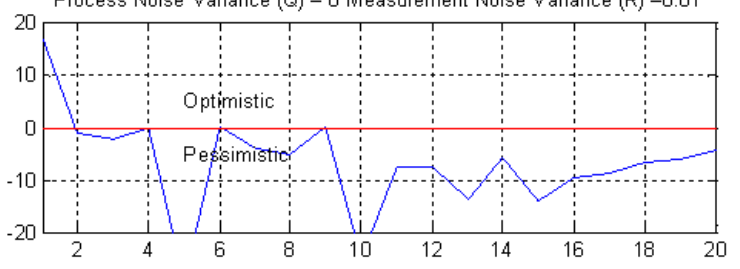

The Average Normalized Estimation Error Squared and 95\% Confidence Interval

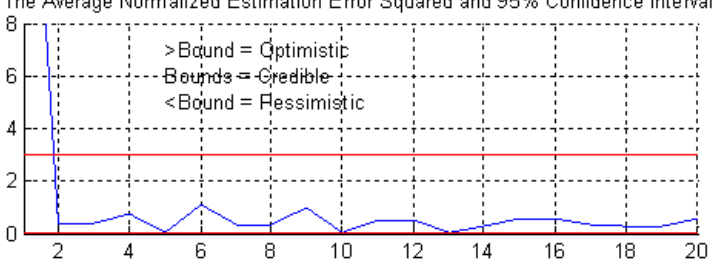

Figure 7. DD1 credibility analysis.

Next, we show performance results to determine a model mismatch or bias in the estimator for the tracking problem using nonlinear methods. Figures 6-8 demonstrate nonlinear tracking using the NCI and ANESS to determine the tracker performance independent of the data.
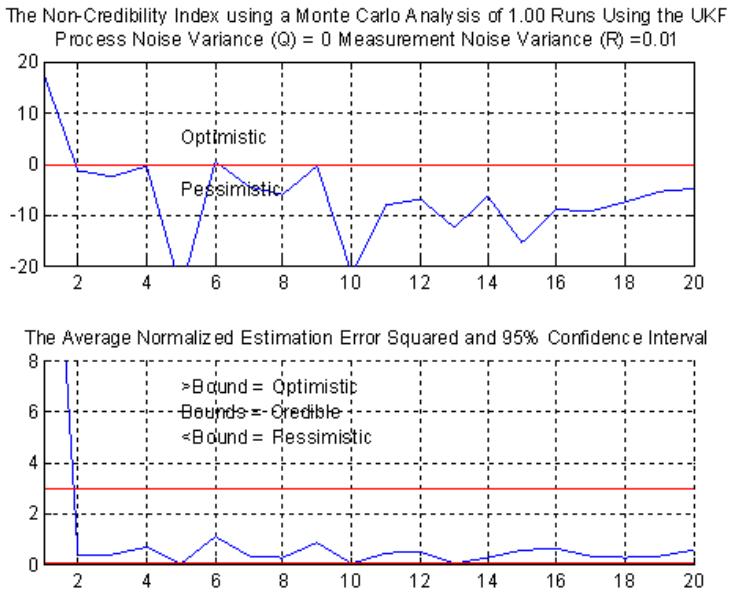

Figure 8. UKF credibility analysis.
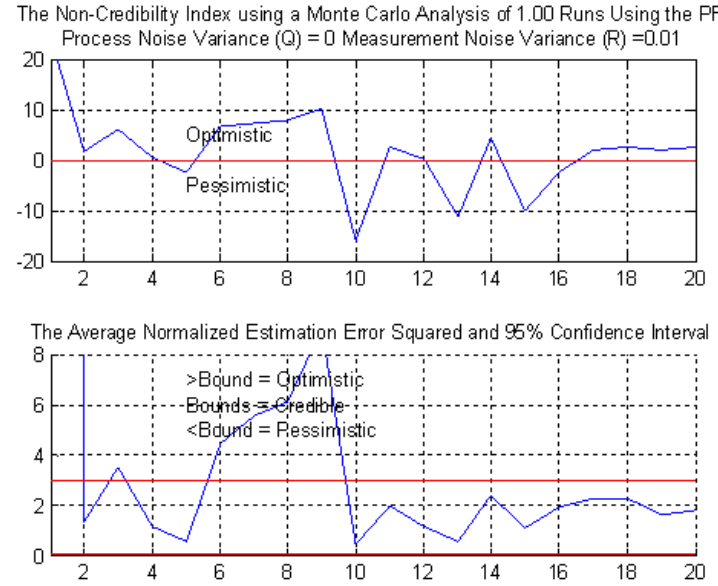

Figure 9. PF credibility analysis.

\section{Discussion \& Conclusions}

We looked at the standard RMS track metrics which show the sensor-tracker accuracy; however, we desired to assess tracker performance independent of the data. To do this, we applied the Relative Track Metrics proposed by X. Rong Li. In a series of simulation experiments, the NCI demonstrated the ability to detect a model mismatch or bias in the results. Also, we were able to implement the $\mathrm{NCI}$ in nonlinear tracking systems. By estimating the particle region, there is an issue with $P F$ re-sampling number versus relative metric analysis in the MSE over estimator covariance. We showed sensitivity metrics that address tracker quality independent of measurements for nonlinear tracker evaluation. Regions of optimism could spawn particles and the regions of pessimism could reduce particles to save computation. Additionally, the NCI and ANESS could control the spread of particles.

Future work will explore (1) the tracker sensitivity metrics to multiple target tracking, (2) target maneuverability, and (3) addressing simultaneous track and identification. These tracker metrics will be developed for a sensor management cost function and user performance validation.

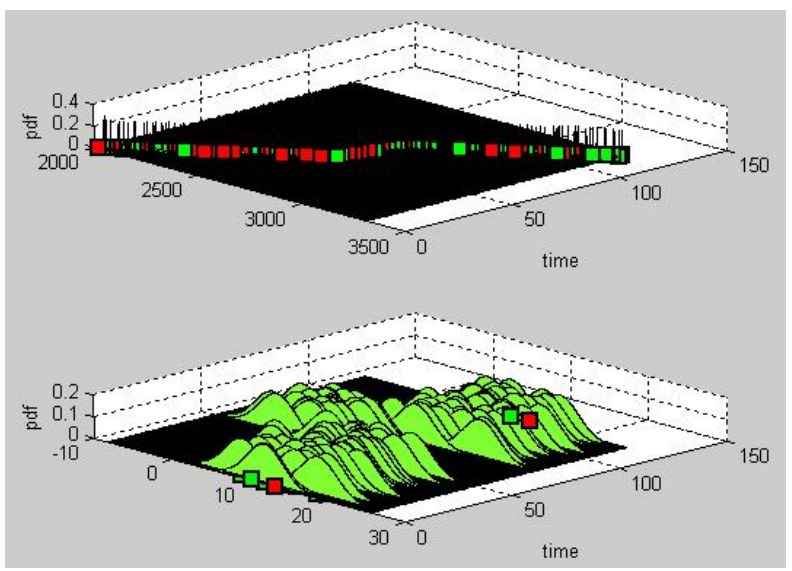

Figure 10. PDF Plot.

\section{References}

[1] X. R. Li \& Z. Zhao, "Evaluation of Estimation Algorithms - Part 1: Local Performance Measures," submitted to IEEE AES, 2004.

[2] P. S. Maybeck, Stochastic models, estimation, and control, Vol. 1, Academic Press, 1979

[3] Y. Bar-Shalom \& X. Li, Multitarget-Multisensor Tracking: Principles and Techniques, YBS, New York, 1995.

[4] E. Blasch, Derivation of A Belief Filter for High Range Resolution Radar Simultaneous Target Tracking and Identification, Ph.D. Dissertation, Wright State University, 1999.

[5] S. Blackman and R. Popoli, Design and Analysis of Modern Tracking Systems, Artech House Publisher, Boston, 1999.

[6] H. A. P Blom \& Y Bar-Shalom, "The interacting Multiple Model algorithm for systems with Markovian Switching coefficients, IEEE Tr. Auto. Cntrl, Vol. 33, 1988, 780-783.

[7] Y. Bar-Shalom, X. R. Li, \& T. Kirubarajan, Estimation with Applications to Tracking and Navigation: Theory, Algorithms and Software, New York Wiley, 2001. 
[8] S. J. Julier, J.K. Uhlmann, \& H. Durrant-Whyte,“ A New approach for filtering Nonlinear Systems, “Amer. Control Conference, 1995.

[9] S. J. Julier, "The scaled Unscented Transformation" American Control Conference, 2002.

[10] M. Mallick, S. Maskell, T. Kirubarajan, \& N. Gordon, "Littoral Tracking using Particle Filter," Fusion2002, pp.935-942.

[11] H. Blom \& E. Bloem, "Joint IMMPDA Particle Filter," Fusion 03.

[12] B. Ristic, S. Arullampalam, \& N. Gordon, Beyond the Kalman Filter: Particle filters for tracking applications, Artech House, 2004.

[13] R. Van der Merwe, Sigma-Point Kalman Filters for Probabilistic Inference in Dynamic State-Space Models, PhD Dissertation, Oregon Health and Service Univ., 2004.

[14] D. Salmond. D. Fisher, \& N. Gordon, "Tracking and Identification for closely spaced objects in clutter," in Proc. European Contr. Conf., Brussels, Belgium, 1997.

[15] E. P. Blasch and L. Hong "Simultaneous Identification and Track Fusion," Conf. on Dec. Control, Orlando, FL, Dec 1998.

[16] E. Blasch, "Data Association through Fusion of Target track and Identification Sets," Fusion 2000, Paris, France, 2000.

[17] E. P. Blasch, "Information-Theory-based feature-aided tracking and identification algorithm for tracking moving and stationary targets through high-turn maneuvers using fusion of SAR and HRR information", Proc. SPIE 4727, 2002.

[18] E Blasch and C Yang, "Ten methods to Fuse GMTI and HRRR Measurements for Joint Tracking and ID", Fusion 04, July 2004

[19] E. Blasch, "Modeling Intent for a target tracking and identification scenario," SPIE 04, 5428, April 2004.

[20] E. Blasch, "Group Tracking, Using Fusion of Simultaneous Track and ID Information", In NATO ASI: Data Fusion for Situation Monitoring, Incident Detection, Alert \& Response Mgt, 2004.

[21] A. Tchamova, J. Dezert, et al, "Target Tracking with Generalized data association based on the general DSm rule of combination," Fusion04, 2004

[22] D. Mušicki, S. Suvorova, S. Challa, "Multi Target Tracking of Ground Targets in Clutter with LMIPDA-IMM", Fusion04, Stockholm, Sweden, June 2004.

[23] D. Angelova \& L. Mihaylova, "Joint Tracking and Classification with Particle Filtering and Mixture Kalman Filtering using Kinematic Radar Information," Digital Signal Processing, 2005.

[24] E. Blasch and B. Kahler, "Multi-resolution EO/IR Tracking and Identification" Fusion 05, July 2005.

Tracking Applications

[25] K. M. Lee, K.M., Z. Zhi, R. Blenis, and E. P. Blasch, "Real-time vision-based tracking control of an unmanned vehicle," IEEE Journal of Mechatronics - Intelligent Motion Control, Oct. 1995.

[26] G. Seetharaman, A. Lakhotia, E. Blasch," Unmanned Vehicles Come of Age: The DARPA Grand Challenge," IEEE Computer Society Magazine, Dec 2006.

[27] E. Blasch, I. Kadar, J. Salerno, M. M. Kokar, S. Das, G. M. Powell, D. D. Corkill, and E. H. Ruspini, "Issues and challenges of knowledge representation and reasoning methods in situation assessment (Level 2 Fusion)", $J$. of Advances in Information Fusion, Dec. 2006.

Recent Tracking Advances

[28] C. Yang and E. Blasch, "Fusion of Tracks with Road Constraints," Advances in Journal for Information Fusion, June 2008.

[29] C. Yang and E. Blasch, "Kalman Filtering with Nonlinear State Constraints", IEEE Transactions AES, Vol. 45, No. 1, Jan. 2009.

[30] H. Chen, G. Chen, E. Blasch, and T. Schuck, " Robust Track Association and Fusion with Extended Feature Matching", invited Chapter in Optimization \& Cooperative Ctrl. Strategies, M.J. Hirsch et al. (Eds.):, LNCIS 381, Springer-Verlag 2009.

[31] T. Wang, Z. Zhu, and E. Blasch, "Bio-Inspired Adaptive Hyperspectral Imaging for real-Time Target Tracking “ IEEE Sensors Journal, Vol. 10, No. 3, pp. 647-654, 2010.

[32] D. Shen, Z. Tian, and E. Blasch, "Information-based Awareness Model and Active Sensing In Sensor Resource Management," IEEE ACC, 2010
[33] H. Ling, L. Bai, E. Blasch, and X. Mei, "Robust Infrared Vehicle Tracking Across Target Change using $\mathrm{L}_{1}$ regularization," Fusion 10, 2010.

[34] K. Palaniappan, F. Bunyak, P. Kumar, I. Ersoy, S. Jaeger, K. Ganguli, A. Haridas, J. Fraser, R. Rao, and G. Seetharaman, "Efficient Feature Extraction and Likelihood Fusion for Vehicle Tracking in Low Frame Rate Airborne Video," Fusion10, 2010.

[35] J. Dezert and B. Pannetier, "A PCR BIMM filter for maneuvering target tracking," Fusion 10, 2010.

Track Performance Evaluation

[36] O.E. Drummond, X. R. Li, and C. He, "Comparison of Various Static Multiple-Model Estimation Algorithms, Proc. SPIE 3373, pp. 510-527, 1998.

[37] O.E. Drummond, "Methodologies for Performance evaluation of Multitarget Multisensor tracking," Proc. SPIE, Vol. 3809, 1999.

[38] R. Rothrock \& O. Drummond, "Performance Metrics for Multiplesensor, Multiple-Target Tracking," Proc. SPIE, Vol. 4048, 2000.

[39] W.D. Blair, "Multitarget Metrics for SAIP Systems," Fusion08.

[40] A. I. El-Fallah, R. P. Mahler, T Zajic, E Sorensen, M. Alford \& R. K. Mehra, "Scientific Performance evaluation for sensor management,“ Proc. SPIE, Vol. 4053, pp. 183-194, 2000.

[41] T. Nguyen, V. Jilkov, and X. R. Li, "Comparison of Sampling-Based Algorithms for Multisensor Distributed Target Tracking, Fusion03.

[42] C.Y. Chong, "Problem Characterization in Tracking/Fusion Algorithm Evaluation," IEEE AES Sys. Mag., July 2001.

[43] X. Zhang \& P. Willet, "Cramér-Rao Bounds for Discrete Time Linear Filtering with Measurement Origin Uncertainties, Tribute to YBS Conf., pp. 546-561. 2001.

[44] M. Hernandez, "Performance bounds for GMTI Tracking," Fusion03, 2003.

[45] J.J. Sudano, \& Jones, M.H., “An Improved Representation of the True Tracking Errors as a Better Alternative to the Kalman Covariance," 2003 Core Tech. for Space Sys. Conf., 2003, CO.

[46] C. Y. Chong and S. Mori, "Metrics for Feature-Aided Track Association," Fusion06, 2006.

[47] J. Dezert, A. Tchamova, F. Smarandache, and P. Konstantinova, "Target Type Tracking with PCR5 and Dempster's rules: A Comparative Analysis," Fusion06, 2006.

[48] E. Blasch, A. Rice, and C. Yang, "Relative Performance Metrics to Determine Model Mismatch," IEEE NAECON, 2008.

[49] R. Schubert, H. Klöden, G. Wanielik, and S. Kälberer, "Performance Evaluation of Multiple Target Tracking in the Absence of Referenced Data, Fusion10, 2010.

Nonlinear Estimation

[50] M. Šimandl, J. Královec, and T. Söderström, "Advanced point-mass method for nonlinear state estimation," Vol. 42, Automatica, 2006

[51] O. Straka and M. Šimandl. "Particle Filter Adaptation based on Efficient Sample Size," System ID Conf., 2006

[52] O. Straka and M. Šimandl. "Adaptive Particle filter with fixed empirical density quality," IFAC System ID Conf., 2008.

[53] O. Straka and M. Šimandl. "Survey of Sample Size Adaption Techniques for Particle Filters," System ID Conf., 2009.

[54] M. Šimandl and J. Duník, "Derivation-Free Estimation Methods: New Results and Performance Analysis," Automatica, March 2009.

[55] M. Šimandl and I. Punčochář, "Active-Fault detection and control: Unified formulation and optimal design," Vol. 45, Automatica, 2009.

[56] O. Straka, M. Flídr, J. Duník, and M. Šimandl, "A software framework and tool for nonlinear state estimation ," System ID Conf., 2009.

[57] O. Straka, M. Flídr, J. Duník, M. Šimandl, and E. P. Blasch, "Nonlinear Estimation Framework in Target Tracking," Fusion10, 2010.

[58] C Yang \& M Miller, "Nonlinear Filtering Techniques for GNSS Data Processing," ION 61st Ann. Mtg/ MITRE Corp. \& Draper Lab., 27-29 June 2005, Cambridge, MA, pp. 690-703.

[59] Doucet, A.; De Freitas, N.; Gordon, N.J. Sequential Monte Carlo Methods in Practice. Springer, 2001. 